\title{
Plantar Grasp Reflex
}

National Cancer Institute

\section{Source}

National Cancer Institute. Plantar Grasp Reflex. NCI Thesaurus. Code C110939.

A newborn reflex that is characterized by flexion of the toes when the sole of the foot is stroked. 